\title{
Sustentabilidad y nanejo agroecológico mediante indicadores en un paisaje agrícola: estudio de caso a nivel de finca, Amazonía Ecuatoriana
}

\section{Sustainability and agro-ecological management through indicators in an agricultural landscape: case study a the farm level, Ecuadorian Amazon}

\author{
Julio Macas $^{1 *}$, Jefferson Morales ${ }^{1}$, Javier Chuquimarca $^{1}$, Betty Reinoso $^{1}$, Boris Soria ${ }^{1}$, Marcela Suigla ${ }^{1}$, Carlos Bravo ${ }^{2}$, Reinaldo Alemán ${ }^{2}$ \\ ${ }^{1}$ Estudiantes de Postgrado, Maestría Agronomía mención en Sistemas Agropecuarios \\ Universidad Estatal Amazónica (UEA), Pastaza 160101, Ecuador. \\ ${ }^{2}$ Docentes Investigadores, Universidad Estatal Amazónica (UEA), Pastaza 160101, Ecuador. \\ "Correspondencia: juliocesarmacas05@gmail.com ; jmoralesharo91@gmail.com ; edgarjavierchuquimarca@gmail.com ; \\ belizamparito94@gmail.com ; bsoriauea@gmail.com ; msuiglauea@gmail.com ; cbravo@uea.edu.ec ; \\ reinaldoap@gmail.com
}

Rec.: 01.06.2019. Acept.: 10.01.2020. Publicado el 30 de junio de 2020

\section{Resumen}

$\mathrm{E}_{\mathrm{s}}^{1}$ objetivo del presente trabajo fue evaluar la sustentabilidad y el manejo agroecológico en la finca el Guayacán, cantón Santa Clara, provincia de Pastaza, Ecuador. Se caracterizó mediante la metodología para evaluar sustentabilidad en la región amazónica ecuatoriana (MESRAE), que involucró 36 indicadores para cuantificar la sustentabilidad se utilizó una escala ordinal y numérica del 1 al 10, considerando niveles de alto ( $>6.5$ a 10$)$, medio $(>5.5$ a 6.5$)$ y bajo $(<5.5)$, distribuidos en cuatro dimensiones, ambiental, socio-cultural, política y económica, con los valores obtenidos para cada dimensión se constituyeron flujogramas tipo "ameba" que permiten visualizar el estado general y nivel de sustentabilidad de la finca, el índice promedio de sustentabilidad presentó el siguiente orden: dimensión ambiental (6.85), dimensión socio-cultural (7.58); dimensión política (4.02); dimensión económica (4.32), los cuales fueron clasificados de alta, media o baja sustentabilidad; el índice obtenido de la finca es 5.69, nivel uno. Los resultados sugieren que la sustentabilidad a nivel de finca presentó puntos críticos en la dimensión ambiental, económica y política. Los indicadores que deben ser mejorados son: a) Nivel de erosión del suelo b) Presencia de insectos benéficos, c) Baja presencia de diversidad biológica d) Ingreso neto mensual de la UPA), e) Costos de insumos agropecuarios (balanceado, desparasitantes, sal), f) Estrategias de comercialización, g) Estrategia de ahorro, h) Programas del estado relacionados con la finca (Sistemas Ganaderos), i) Nivel de aceptación de los programas del estado, j) Articulación de la finca con otras instituciones acompañantes.

Palabras clave: Agroecología, sostenibilidad, resiliencia.
$T^{1}$ The objective of this work was to evaluate sustainability and agro-ecologicalmanagementinthe Guayacán estate, Santa Clara canton, Pastaza province, Ecuador. It was characterized by the methodology for assessing sustainability in the Ecuadorian Amazon region (MESRAE), which involved 36 indicators to quantify sustainability an ordinal and numerical scale of 1 to 10 was used, considering high levels ( $>6.5$ to 10 ), medium ( $>5.5$ to 6.5$)$ and low $(<5.5)$, distributed in four dimensions, environmental, socio-cultural, political and economic, with the values obtained for each dimension were constituted "ameba" flowcharts that allow to visualize the general state and level of sustainability of the farm, the average sustainability index presented the following order: environmental dimension (6.85), socio-cultural dimension (7.58); political dimension (4.02); economic dimension (4.32), which were classified as high, medium or low sustainability; the index obtained from the farm is 5.69 , level one. The results suggest that sustainability at the farm level presented critical points in the environmental, economic and political dimension. Indicators to be improved are: a) Soil erosion level b) Presence of benzephic insects, c) Low presence of biodiversity d) Monthly net income of UPA), e) Agricultural input costs (balanced, deparasive, salt), f) Marketing strategies, g) Savings strategy, h) State programs related to the farm (Ganader Systems), i) Level of acceptance of state programs, j) Articulation of the farm with other accompanying institutions.

Key words: Agroecology, sustainability, resilience. 


\section{Introducción}

L a Región Amazónica Ecuatoriana (RAE) comprende el 2\% de la cuenca del río Amazonas, su extensión es $116441 \mathrm{~km}^{2}$ y representa la región natural más grande (47\%) del Ecuador, los recursos amazónicos necesitan ser manejados y conservados con criterios de sustentabilidad (Bravo et al., 2017).

Según, Nieto y Caicedo (2012), la situación social rural de la región amazónica, posee bajos ingresos, su actividad económica se basa principalmente a las actividades agropecuarias. Sin embargo, su clima extremadamente lluvioso, con suelos poco fértiles, susceptibles a erosión, explicarían la poca aptitud de la región para actividades agropecuarias convencionales, pero aptos para sistemas productivos análogos al bosque; así como lo menciona, Rivadeneira y Cervantes (2015), se deben, incorporar técnicas e innovaciones, basadas en principios ecológicos y en un alto conocimiento de los suelos, la nutrición de las plantas, del manejo de plagas, del potencial genético de los cultivos y animales y de las interacciones biológicas y ecológicas.

Ciertamente la agroecología es lograr una agricultura sostenible a partir del diseño y manejo de sistemas de producción con seres vivos sostenibles, al igual que el diseño de estrategias para que agroecosistemas insostenibles alcancen la sostenibilidad y la conversión agroecológica (Echeverri et al., 2017). Según Altieri (2010) la agroecología es "una agricultura socialmente justa, económicamente viable y ambientalmente sana".

Según Altieri y Nicholls, (2013), la combinación de prácticas tradicionales de los sistemas de manejo y estrategias agroecológicas puede constituir una ruta factible para incrementar la productividad, la sustentabilidad y la resiliencia de los sistemas socioecológicos. Se han desarrollado y sistematizado marcos metodológicos para su aplicación a nivel de fincas, que permiten medir su desempeño ambiental, social, económico y político, es decir la evaluación de la sustentabilidad en un contexto de pequeños agricultores y ganaderos, mediante la selección participativa de indicadores (Bravo et al., 2017).

Bajo este marco de referencia el objetivo del presente estudio fue: caracterizar la sustentabilidad a nivel de finca mediante indicadores de las dimensiones: ambiental, sociocultural, económico y política.

\section{Materiales y métodos}

$\mathrm{E}$ 1 presente estudio se realizó en la finca El Guayacán, ubicada en el km 47 vías Puyo - Tena, sector Rey del Oriente, cantón Santa Clara, provincia de Pastaza (Figura 1). La altura oscila entre 500 a $600 \mathrm{msnm}$, con una temperatura promedio anual de $25{ }^{\circ} \mathrm{C}$. El área de estudio se clasifica como Bosque Húmedo Tropical, con precipitaciones promedio de $4.400 \mathrm{~mm} /$ año y una alta humedad relativa de entre 87 y $89 \%$ (Uvidia et al., 2015).

Para realizar la caracterización de la sustentabilidad, se aplicó la metodología llamada metodología para evaluar la sustentabilidad en la región amazónica ecuatoriana (MESRAE) propuesta por Bravo et al., (2017), que consistió en el análisis de 36 indicadores. Para cuantificar la sustentabilidad se utilizará una escala ordinal y numérica del 1 al 10 , considerando niveles de alto $(>6.5$ a 10$)$, medio $(>5.5$ a 6,5$)$ y bajo $(<5.5)$, distribuidos en las dimensiones ambientales, socioculturales, económicas y políticas.

La encuesta fue realizada al Sr. Dionisio Calapucha, hijo mayor de la familia Calapucha Vargas, en un conversatorio de tres horas, en la Finca El Guayacán, sector Rey de Oriente del cantón Santa Clara, provincia de Pastaza.

La encuesta tiene las siguientes secciones: 1) componentes agrícola y animal con sus respectivas prácticas de manejo e insumos, 2) componente familiar, 3) aspectos socio- culturales, económicos y políticos, utilizados para la sistematización y valoración de los indicadores, evidenciando la situación actual mediante la elaboración de un flujo diagrama de la finca.

\section{Sistematización de la información recolectada $y$ valoración de los indicadores}

La valoración de cada indicador se determinó mediante seis diferentes criterios en una escala ordinal y numérica del 1 al 10, considerando niveles de alto ( $>6.5$ a 10), medio (>5.5 a 6.5) y bajo $(<5.5)$. Para la selección de los puntos críticos dentro de cada dimensión se tomó como criterio aquellos indicadores que mostraron una valoración menor de 5.5. Se calculó el promedio de cada uno de los indicadores a partir de la valoración dada y posteriormente el promedio general de las dimensiones, para identificar el índice potencial de sustentabilidad de la finca.

\section{Diagrama tipo AMEBA}

Se realizó una representación gráfica tipo AMEBA de los indicadores por dimensión, utilizando Microsoft Excel, metodología conocida como Marco MESMIS quien también recomienda el diagrama "ameba" ésta permite contrastar los valores reales de los indicadores con los valores ideales (López, et al., 2001). Se considera que cuanto más se aproxime el valor a 10 más sustentable el indicador y por el contrario cuando se encuentran por debajo de 5.5 no se considera sustentable ayudando de esta manera a priorizar el tipo de intervención necesaria para corregir, planificar e incorporar estrategias que fortalezcan el perfil ambiental, social, económico y político de la unidad de manejo. 


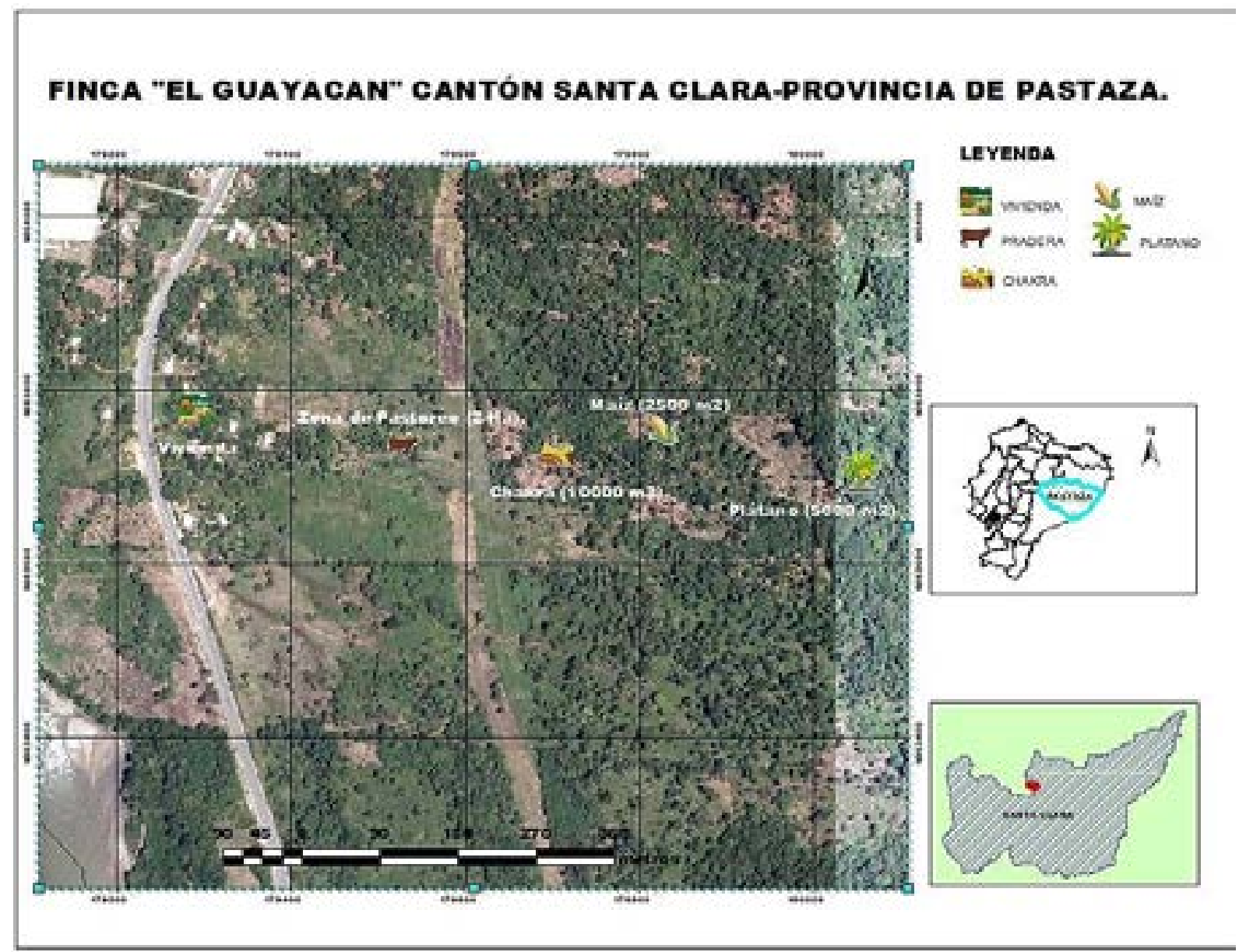

Figura 1. Localización de la finca “El Guayacán” en el cantón Santa Clara, provincia de Pastaza

\section{Resultados y discusión}

$\mathrm{L}$ os sub sistemas resultantes son: familiar, vegetal y agroforestal suelo y animal; las entradas son el resultado de la comercialización de la producción agropecuaria, y las salidas son los recursos necesarios de adquirir para cumplir con los procesos productivos; con las relaciones funcionales de la finca, su entorno ambiental y social se construyó el modelo sistémico actual.

\section{Flujograma de diagnóstico}

La Figura 2, representa el diagnóstico, la situación actual de la sustentabilidad del sistema agroproductivo establecido en la finca El Guayacán determina las entradas, salidas y las interacciones entre sus componentes agroproductivas basada en tres aspectos.

a) Definición de los límites espaciales; La finca tiene una extensión de 21 ha, se identificaron cuatro sub sistemas: 1) familiar, 2) vegetal y agroforestal, 3) suelo y 4) animal, con una participación en extensión del
$4.76 \%, 9.52 \%, 23.80 \%$ y $61.88 \%$, respectivamente. El subsistema familiar (1 ha) consiste en la infraestructura de la vivienda, establo, gallineros y porquerizas. El subsistema vegetal está conformado por el cultivo de maíz $(0.25$ ha, $1.19 \%)$, plátano $(0.5$ ha, $2.38 \%)$, yuca y plátano $(0.25$ ha, $1.19 \%)$, yuca $(0.25$ ha, $1.19 \%)$ cacao (1 ha, $4.76 \%$ ), café ( 1 ha, $4.76 \%$ ), chacra ( 1 ha, $4.76 \%$ ). El subsistema animal contempla 13 ha, representan el $61.88 \%$, el subsistema suelo, compuesto por 3 ha de bosque secundario (14.28\%), bosque primario 1 ha $(4.76 \%)$, ríos y quebradas 1 ha $(4.76 \%)$.

\section{b) Identificación de subsistemas y su interrelación;} Las interrelaciones entre los distintos subsistemas y su influencia en el propósito principal del agro ecosistema pueden caracterizarse mediante los distintos tipos de relaciones tales como: De cadena directa: donde el subsistema cultivo (pasto miel, Setaria splendida) es utilizado para alimentar al subsistema pecuario (bovino, avícola) y éste a su vez produce derivados tales como leche, huevos y proteína animal para el subsistema familiar y venta a mercados (centros de acopio). La 
a) producción del cultivo de maíz como fuente principal de alimentación a las aves, producen carne y huevos para la familia fomentando el ingreso económico para la familia, por venta de sobrante de maíz y aves de descarte. Los recursos fitogenéticos como el barbasco $\mathrm{y}$ hojas de bijao presentan un potencial mercado agroecológico en el control integrado de plagas, al producir insecticidas biológicos.

De cadena cíclica: La retroalimentación de los residuos de cobertura o biomasa que aporta el pasto una vez que termina su consumo, los cuales son descompuestos o incorporados al suelo. De cadena competitiva: el subsistema forestal puede competir con el subsistema cultivo (pasto miel, Setaria splendida) por luz, aguas, nutrientes entre otras entradas. Estos dos sistemas pueden contrastarse, donde el sistema cultivo compite con el forestal o bosque por el avance de la frontera agrícola.

c) La relación entre el agro ecosistema, su entorno social y ambiental; La conexión del agroecosistema con el mercado se puede ejemplificar de dos maneras: 1) venta del producto (ferias, mataderos y centros de acopio) Por ejemplo, los principales productos comercializados que caracterizan estos agro ecosistemas: ventas de animales para carne y la producción de leche, huevos y sus derivados (queso), así como la parte agrícola-forestal cuyos beneficios están enfocados a los remanentes de producción de los cultivos principales (maíz, plátano, yuca, café, cacao), así como de los secundarios la madera, barbasco y bijao permiten un ingreso económico familiar y 2) lugar donde se adquiere los insumos agrícolas como: balanceado, sal mineralizada, medicinas, vitaminas plaguicidas, son adquiridos en las casas comerciales que no necesariamente emiten un criterio técnico en sus recomendaciones, generando en el agricultor el incremento de sus costos de producción.

La vinculación directa con el ambiente se caracteriza por la valorización del conocimiento ancestral establecidos en la finca y la conservación del bosque primario, así mismo minimizando el uso de agroquímicos permitiendo la conservación del recurso suelo, manteniendo la dinámica de la macro y micro fauna y su actividad biológica. El ejercicio de

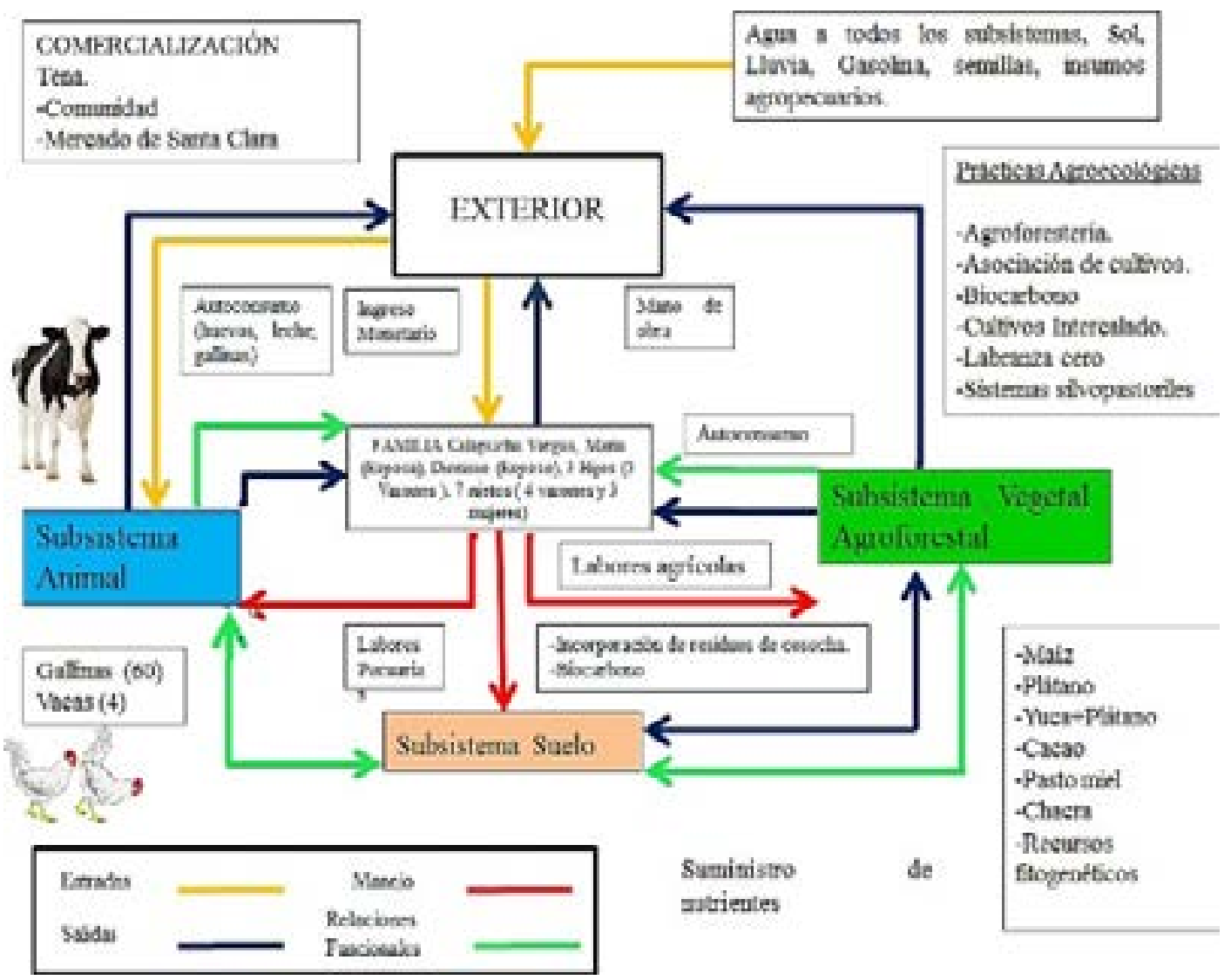

Figura 2. Flujograma de diagnóstico de la finca el Guayacán 
producción denota varias interacciones positivas entre los subsistemas endógenos familiares y exógenos de mercado. Estas interacciones generan dentro del predio un índice de 5.69 de sustentabilidad.

\section{Sistematización de resultados por dimensión}

Una vez analizados los 36 indicadores de sustentabilidad, doce son para la dimensión ambiental y sociocultural, ocho para la económica y cuatro para político se cuantificará mediante la escala ordinal y numérica del 1 al 10, considerando niveles de alto $(>6.5$ a 10$)$, medio $(>5.5$ a 6.5$)$ y bajo $(<5.5)$

\section{Dimensión ambiental}

En la figura 3, se observó cómo puntos críticos el nivel de erosión del suelo, la baja presencia de insectos benéficos y baja presencia de la actividad biológica con valores inferiores a 5.5 en promedio representa la situación actual del sistema agro productivo establecido en la finca El Guayacán donde se evidencia los componentes que integran cada uno de los subsistemas, los cuales interactúan como medios de subsistencia para los miembros de la finca. Independientemente de la finca las propiedades físicas (color, textura, estructura granular, porosidad) y el contenido de materia orgánica de los suelos son las variables de mayor contribución a la fertilidad integral, surgiendo una adecuada condición física y biológica, que ayuda a la penetración y desarrollo de raíces, favoreciendo el aprovechamiento de nutrientes y agua en el suelo (Power, 2010; Bravo et al., 2015). Los resultados confirman la importancia que tiene la materia orgánica sobre la calidad física del suelo, el reciclaje de nutrientes como fuente principal de nitrógeno y otros elementos en los ecosistemas y agroecosistemas amazónicos (Bravo el al., 2015). El proceso de erosión se ve magnificado particularmente con pendientes (10-35\%), la baja profundidad efectiva del suelo y las

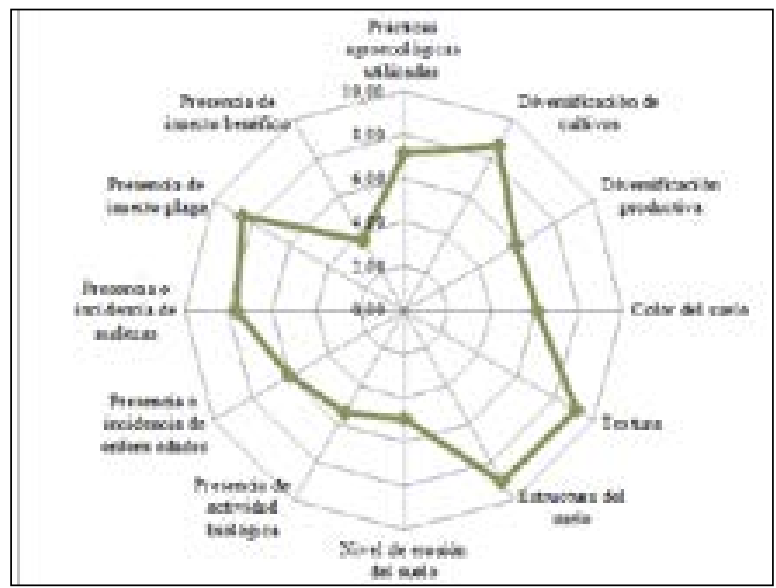

Figura 3. Representación ameba de los indicadores de la dimensión ambiental de la finca El guayacán

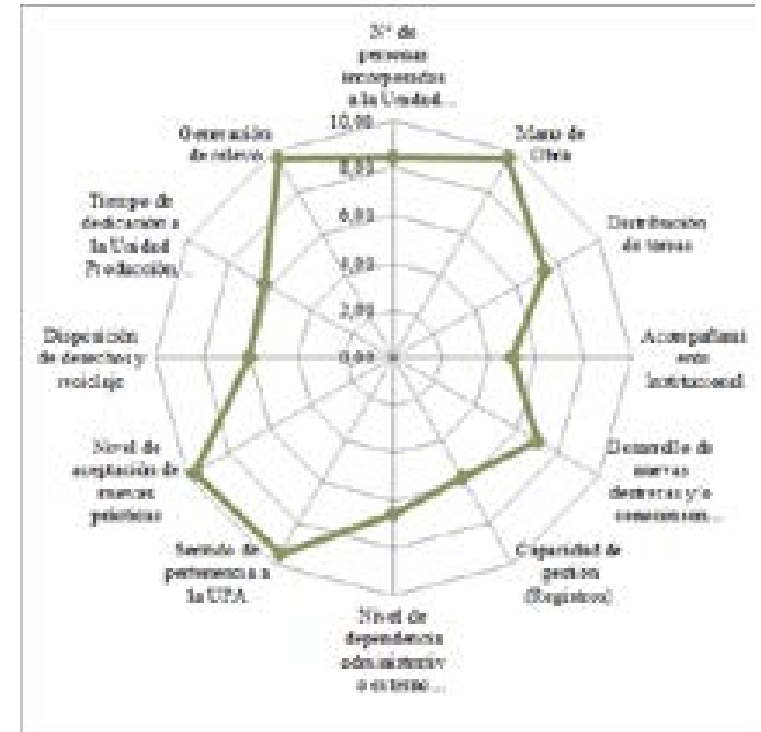

Figura 4. Representación ameba de los indicadores de la dimensión socio - cultural de la UPA el Guayacán

condiciones climáticas de la zona caracterizada por altas e intensas precipitaciones durante todo el año, que dan como resultado una estructura simplificada del paisaje (Vargas et al., 2014). Estudios similares de evaluación de sustentabilidad en la provincia de Pastaza, presentan valores en el sistema ambiental entre 5.41 y 7.63, concluyendo que la mayoría de las fincas, presentaron un índice de sustentabilidad de bajo a moderado, sugiriendo que gran parte de los indicadores y en todas las dimensiones muestran niveles críticos que necesitan ser mejorados (Bravo et al., 2017).

\section{Dimensión socio - cultural}

En la Figura 4, se observó la inexistencia de puntos críticos, los valores se encontraron entre el nivel medio y alto. Estudios de sustentabilidad realizados en diferentes fincas de cantones Pastaza y Arosemena Tola Bravo et al., (2018) y Bravo et al., (2017), muestran que el indicador socio-cultural es muy oscilante, presentando valores 4.57 y 7.86 clasificado como bajas, moderadas o altas, la finca en estudio presentó un valor de 7.8 demostrando que mantiene un sólido componente familiar demostrando fortalezas tales como: tiempo de dedicación a la finca, sentido de pertenencia y nivel de aceptación de nuevas prácticas (Bravo et al., 2017).

\section{Dimensión económica}

En la Figura 5 se observa como puntos críticos el ingreso neto mensual de la finca, los costos de insumos agropecuarios, estrategias de comercialización y estrategias de ahorro con valores inferiores a 5.5.

Los valores y todos los indicadores económicos en la finca están asociados a un modelo de sistema 
que no integra distintas actividades productivas ni la transformación del producto primario para aportar valor agregado lo cual le confiere baja sustentabilidad y bajo desempeño económico. Los bajos ingresos están relacionados con niveles de pobreza existentes en el medio rural (Nieto y Caicedo, 2012), manejo inadecuado de las fincas, altos costos de producción con poco nivel tecnológico y débil base agroecológica (Vargas et al., 2014).

\section{Dimensión político}

En la Figura 6, se evidencia como puntos críticos los programas del estado relacionados con la finca, el nivel de aceptación de los programas del estado y la articulación de la finca con otras instituciones acompañantes con valores inferiores a 5.5. Se percibe que los programas antes mencionados no están vinculados en cooperación interinstitucional, situación que hace que el recurso invertido no llegue a todos, dejen de ser eficiente $\mathrm{y}$ no se conviertan en motores de desarrollo regional. A diferencia de Bravo et al., (2017) quien menciona que el agricultor no se siente comprometido con las mesas técnicas considerándolas como pérdida de tiempo, donde no se consiguen resultados concretos, el productor de la finca el Guayacán tiene características de liderazgo aprovechando cada uno de estos beneficios, generando un nivel medio en sustentabilidad.

En la Figura 7, se evidencia las dimensiones críticas que afectan el nivel de sustentabilidad de la finca. Al igual que la investigación realizada por Bravo et al., (2017) en la cual involucra la evaluación de la sustentabilidad mediante indicadores de producción, los resultados sugieren que la sustentabilidad a nivel finca presentan niveles críticos en cada dimensión, siendo la política y económica la de mayores limitaciones. De esta manera se demuestra que la sustentabilidad no solo depende de aspectos técnicos y ambientales sino también de

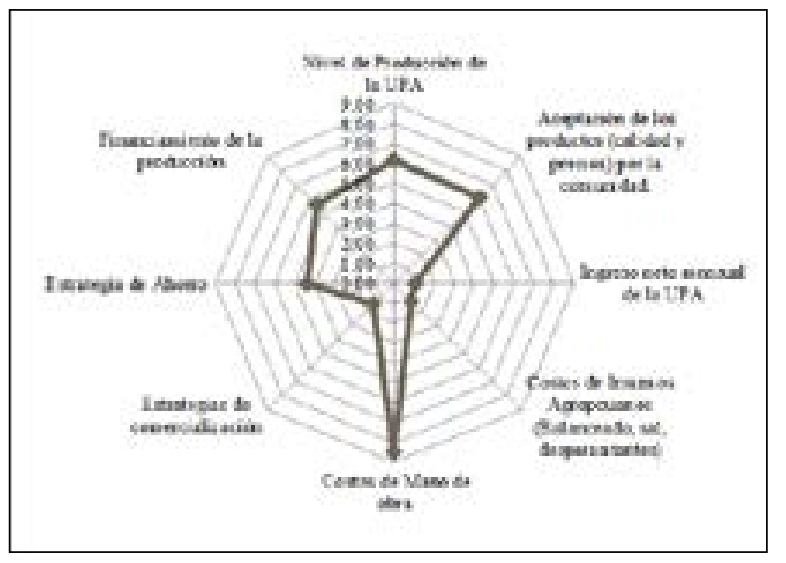

Figura 5. Representación ameba de los indicadores de la dimensión económica de la finca el Guayacán

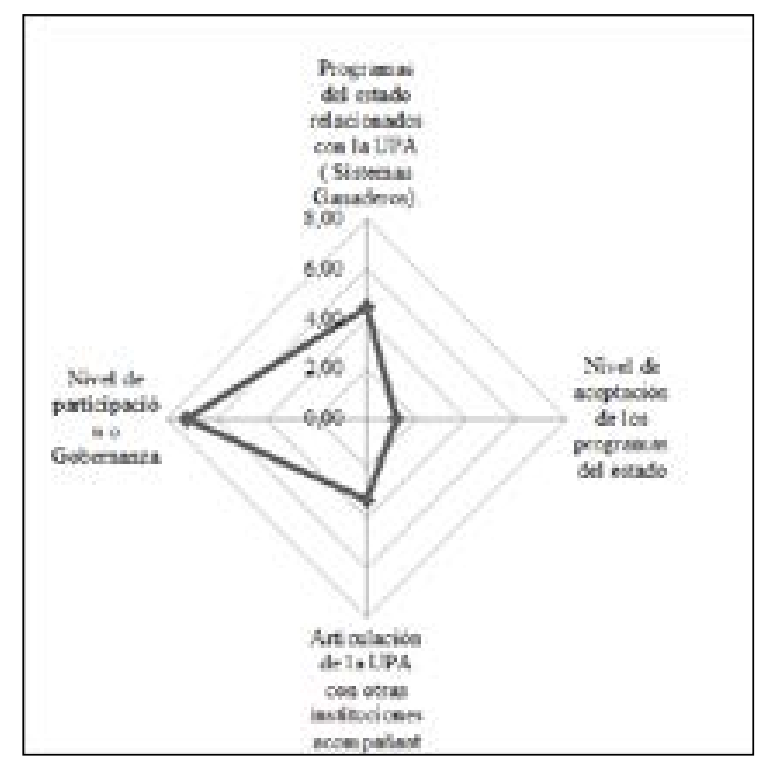

Figura 6. Representación ameba de los indicadores de la dimensión política de la UPA el Guayacán

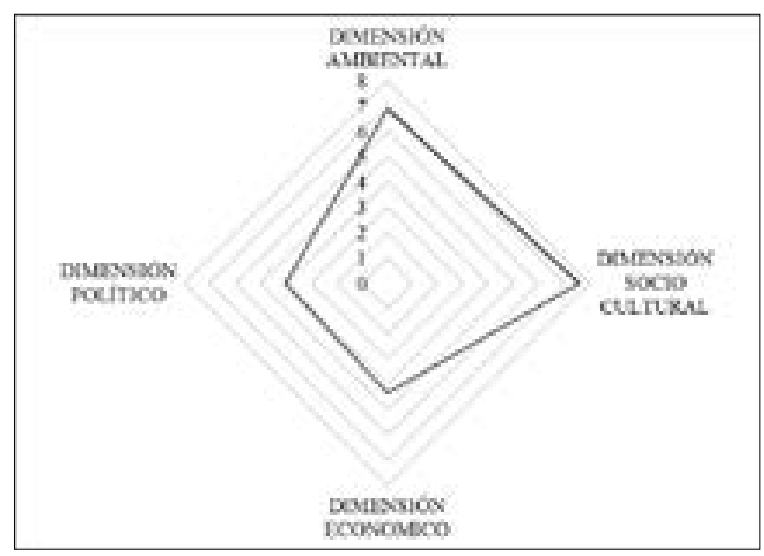

Figura 7. Representación ameba de los indicadores de las cuatro dimensiones de la finca el Guayacán

elementos socioculturales, económicos y políticos los cuales representan los factores más limitantes en la finca.

\section{Determinación de puntos críticos}

En el Cuadro 1 se presentan los valores promedios de los indicadores críticos en la dimensión ambiental, económica y política de la finca. Estos valores son considerados bajos y afectan directamente el nivel de sustentabilidad de la finca.

\section{Recomendaciones de los puntos críticos resultantes del diagnóstico de la finca}

En los Cuadros 2, 3 y 4 se presentan las recomendaciones en función de los puntos críticos identificados en cada dimensión: 
Cuadro 1. Valoración de los puntos críticos en función de los indicadores y las dimensiones de la finca el Guayacán

\begin{tabular}{|c|c|c|}
\hline Dimensiones & Indicadores & $\begin{array}{l}\text { Calificación } \\
\text { promedio }\end{array}$ \\
\hline \multirow{3}{*}{ Componente ambiental } & Nivel de erosión del suelo & 4.92 \\
\hline & Presencia de insecto benéfico & 3.70 \\
\hline & Baja presencia de actividad biológica & 5.38 \\
\hline \multirow{4}{*}{ Componente económico } & Ingreso neto mensual de la finca & 1.08 \\
\hline & $\begin{array}{l}\text { Costos de Insumos Agropecuarios (Balanceado. } \\
\text { sal. desparasitantes) }\end{array}$ & 1.23 \\
\hline & Estrategias de comercialización & 1.50 \\
\hline & Estrategia de Ahorro & 4.42 \\
\hline \multirow{3}{*}{ Componente político } & $\begin{array}{l}\text { Programas del estado relacionados con la finca } \\
\text { (Sistemas Ganaderos). }\end{array}$ & 4.45 \\
\hline & Nivel de aceptación de los programas del estado & 1.17 \\
\hline & $\begin{array}{l}\text { Articulación de la finca con otras instituciones } \\
\text { acompañantes }\end{array}$ & 3.25 \\
\hline
\end{tabular}

Cuadro 2. Recomendaciones de los puntos críticos en función de la dimensión ambiental de la finca el Guayacán

Punto crítico suelo

\section{Recomendación}

- $\quad$ Siembra en curvas de nivel.

- $\quad$ Uso de cobertura como maní forrajero y especies adaptadas al sector

- $\quad$ Siembra en contra de la pendiente.

- $\quad$ Construcción de terrazas.

- Sembrar cultivos de periferia como hierba luisa, melina, laurel, caña guadua, entre otras.

- $\quad$ Siembra de plantas nectaríferas Cassia reticulata, Tabaquilla Borredia laevis, Urena lovata

- $\quad$ Disminución y adecuado manejo del uso de plaguicidas.

Baja presencia de insecto benéfico

Baja presencia de actividad biológica
- Incorporación de materia orgánica en los planes de fertilización de los cultivos

- $\quad$ Disminución de uso indiscriminado de agroquímicos.

- $\quad$ Rotación y diversificar de cultivos con leguminosas. 
Macas et al., 2020

Cuadro 3. Recomendaciones de los puntos críticos en función de la dimensión económica de la finca el Guayacán.

\begin{tabular}{|c|c|}
\hline Punto crítico & Recomendación \\
\hline $\begin{array}{l}\text { Bajo ingreso neto } \\
\text { mensual de la UPA }\end{array}$ & $\begin{array}{ll}\text { - } & \text { Implementación de porcinocultura. } \\
\text { - } & \text { Aumentar la extensión de producción. } \\
\text { - } & \text { Producción de miel. } \\
\text { - } & \text { Implementación de producción piscícola. } \\
\text { - } & \text { Implementación del cultivo de maíz asociado. }\end{array}$ \\
\hline $\begin{array}{l}\text { Alto costo de insumos } \\
\text { agropecuarios }\end{array}$ & $\begin{array}{l}\text { - } \quad \begin{array}{l}\text { Reconocimiento de plantas endémicas y capacitación en la producción de } \\
\text { bio-insumos. } \\
\text { - }\end{array} \text { Acercamiento con instituciones públicas para beneficiarse de programas } \\
\text { con subsidios. } \\
\text { - Manejo integrado de plagas. }\end{array}$ \\
\hline $\begin{array}{l}\text { Estrategias de } \\
\text { comunicación }\end{array}$ & $\begin{array}{ll}\text { - } & \text { Exploración de nuevos mercados. } \\
\text { - } & \text { Revalorización de los productos de la UPA. } \\
\text { - } & \text { Aprovechamiento de los recursos existentes para realizar agroturismo. } \\
\text { - } & \text { Creación de un punto de venta. } \\
\text { - } & \text { Pesca deportiva en su finca- }\end{array}$ \\
\hline Estrategias de ahorros & $\begin{array}{l}\text { - Mejorar la calidad y cantidad de espacio por área de superficie. } \\
\text { - } \quad \text { Buscar fuentes de financiamiento de bajo interés y a largo plazo para la } \\
\text { adquisición de áreas de tierra. }\end{array}$ \\
\hline
\end{tabular}

Cuadro 4. Recomendaciones de los puntos críticos en función de la dimensión política de la finca el Guayacán.

\begin{tabular}{|c|c|}
\hline Punto crítico & Recomendación \\
\hline \multirow{3}{*}{$\begin{array}{l}\text { Programas del estado } \\
\text { relacionado con la UPA }\end{array}$} & $\begin{array}{l}\text { - Buscar un acercamiento institucional, participando en talleres y llevándolo a } \\
\text { la práctica, seguimiento de resultados. }\end{array}$ \\
\hline & $\begin{array}{l}\text { - Charlas informativas sobre los programas de incentivos (créditos, uso de } \\
\text { biocontroladores, valor agregado a los productos) }\end{array}$ \\
\hline & - Acercamientos con organizaciones no gubernamentales. \\
\hline \multirow{2}{*}{$\begin{array}{l}\text { Nivel de aceptación de los } \\
\text { Programas del estado }\end{array}$} & $\begin{array}{l}\text { - Búsqueda de instituciones serias y no politizadas, comprometidas con el agro } \\
\text { para satisfacer la necesidad de la UPA. (MAG, INIAP, AGROCALIDAD.) }\end{array}$ \\
\hline & $\begin{array}{l}\text { - Reforzar el vínculo del agricultor con el CIPCA a través del acompañamiento } \\
\text { y seguimiento técnico en la inclusión de prácticas amigables con el ambiente. }\end{array}$ \\
\hline \multirow{3}{*}{$\begin{array}{l}\text { Articulación de la UPA } \\
\text { con otras instituciones } \\
\text { acompañantes }\end{array}$} & $\begin{array}{l}\text { - Rediseño del sistema productivo en la UPA para una mejor visualización y } \\
\text { apreciación del conocimiento local con el técnico. }\end{array}$ \\
\hline & $\begin{array}{l}\text { - Creación de redes de actores (agricultores-instituciones) para propiciar el } \\
\text { intercambio de conocimiento. }\end{array}$ \\
\hline & $\begin{array}{l}\text { - Participación en intercambios de experiencias con asociaciones de agricultores } \\
\text { cuyo manejo de la UPA sea bajo enfoque agroecológico. }\end{array}$ \\
\hline
\end{tabular}




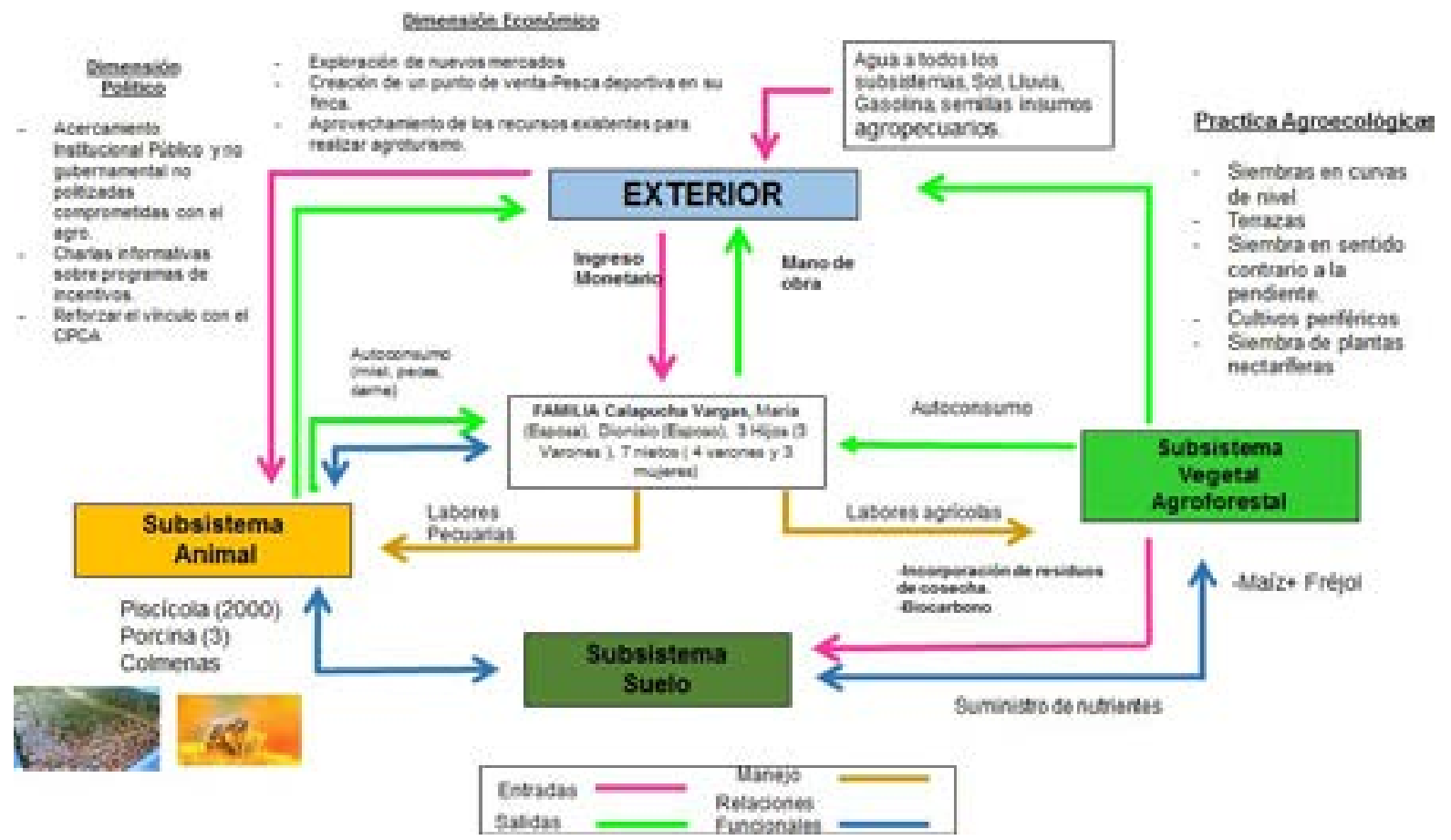

Figura 8. Flujo diagrama con las nuevas relaciones propuestas para el sistema de producción

Flujograma la propuesta para el incremento de la sustentabilidad de la UPA el Guayacán.

Considerando los puntos críticos producto de la valoración en la caracterización de la finca el Guayacán y la forma como contribuyen o no a la eficiencia de los sistemas productivos y a la conservación ambiental se realiza la siguiente propuesta de manejo agroecológico (Figura 8).

Mediante la propuesta de alternativas de manejo agroecológico se pretende incrementar el nivel de sustentabilidad de la UPA a una categoría media, con valores promedios de 7 , permitiendo tener un nivel de sustentabilidad y una conversión paulatina y positiva de la UPA a un largo plazo.

\section{Conclusiones}

Namb determinaron puntos críticos en el componente ambiental: erosión del suelo y baja presencia de insectos benéficos. En el componente económico: ingresos económicos, costos de insumos agropecuarios, estrategias de comercialización y estrategia de ahorro. En el componente político: programas de estado, nivel de aceptación de programas de estado y articulación de estado.

La finca El Guayacán presentó un índice agroecológico de 5.69 considerado un nivel medio muy cercano al nivel bajo, sin embargo se necesita enfatizar en los puntos críticos para fortalecer el proceso de conversión agroecológica, mediante dicha ejecución se pretende alcanzar en un plazo de 12 meses un índice agroecológico de 6.34 un nivel medio aceptable de sustentabilidad. El proceso de transición y fortalecimiento en este nivel de producción agrícola es importante considerar que la producción agrícola puede ir de la mano agroecología, mediante la ejecución de planes y estrategias que permitan al agricultor ser productivo y amigable con el ambiente.

Se recomienda ejecutar un plan de acción que permita el desarrollo de las propuestas planteadas, en una línea del tiempo de doce meses donde se evidencie la aplicabilidad al menos el 33\% trimestralmente.

Generar asociaciones con productores agropecuarios vecinos que les permita la representatividad ante empresas públicas, entidad académica, organizaciones no gubernamentales e instituciones de investigación y desarrollo del estado.

\section{Bibliografia}

Altieri, M. A., \& Nicholls, C. I. (2013). Agroecología y resiliencia al cambio climático: principios y consideraciones metodológicas. Revista de Agroecología, 8(1), 7-20.

Altieri, M.A., 2010. ¿Por qué la agricultura campesina? Agroecología, movimientos sociales y políticas a favor de la soberanía alimentaria, in: Gascón, J., Montagut, X. (Eds.), ¿Cambio de Rumbo En Las Políticas Agrarias 
Latinoamericanas? Estado, Movimientos Sociales Campesinos Y Soberanía Alimentaria.

Bravo, C; Marín, H; Marreno, P; Ruiz, M; Torres, B; Navarrete, H; Durazno, G; Changoluisa, G; (2017). Evaluación de la sustentabilidad mediante indicadores en unidades de producción de la Provincia de Napo, Amazonía Ecuatoriana, Bioagro, Volúmen 29

Bravo, C; Benítez, D; Vargas, J; Alemán, R; Torres, B; Marín, H; (2015). Caracterización socio-ambiental de unidades de producción agropecuaria en la Región Amazónica

Ecuatoriana: Caso Pastaza y Napo. Revista Amazónica Ciencia y Tecnología, Volumen 4 №1- (Pag 3-31)

Bravo, C; Aleman, R; Marin, H, Chimborazo, Cy Navarrete, $\mathrm{H}$. (2018). Potencial de sustentabilidad en agroecosistemas ganaderos de la región amazónica ecuatoriana. VI Congreso Latinoamaericano X Congreso Brasileiro V seminario DO DF E Entorno. Brasilia - DF, Brasil

Echeverri, G., Fernando, L., Ríos Osorio, L., Durán, E., Luisa, M., Echeverri, G., Luisa, M. (2017). Proposal of some general principles for the science of agroecology: a reflection. Revista Lasallista de Investigación, 14(2), 212-219. https://doi.org/10.22507/rli.v14n2a20

Nieto C., C., y Caicedo V., C. (2012). Análisis reflexivo sobre el desarrollo agropecuario sostenible en la amazonia ecuatoriana. Joya de los Sachas, Ecuador: INIAP, Estación Experimental Central de la Amazonia. (Publicación Miscelánea no. 405).

López, S., Masera, O. y Astier, M. (2001, abril). Evaluando la sostenibilidad de los sistemas agrícolas integrados: El Marco MESMIS. Boletín ILEIA, 25-27.

Power, A. 2010. Ecosystem services and agriculture: tradeoff and synergies. Phil. Trans. R. Soc. B. 365: 2959-2971.

Rivadeneira Cervantes, M. B. (2015). Agroecología: Aportes para la Conservación de los Recursos Naturales.

Uvidia, H., J.L. Ramírez, I. Leonard, J.C. Vargas, D. Verdecia y M. Andino. 2015. Inventario de la sucesión vegetal secundaria en la provincia de Pastaza, Ecuador. Revista Electrónica de Veterinaria 16(11): 1-8.

Vargas-Burgos, J.C., D. Benítez, V. Torres, S. Ríos, H. Navarrete y D. Pardo. 2014. Tipificación de las fincas ganaderas de doble propósito en la provincia de Pastaza. Revista Amazónica Ciencia y Tecnología 3. 183-197. 